\title{
Distributed Variable-Baseline Stereo SLAM from two UAVs
}

\section{Conference Paper}

Author(s):

Karrer, Marco; Chli, Margarita (1)

Publication date:

2021

Permanent link:

https://doi.org/10.3929/ethz-b-000484309

Rights / license:

In Copyright - Non-Commercial Use Permitted

Originally published in:

https://doi.org/10.1109//CRA48506.2021.9560944

\section{Funding acknowledgement:}

183720 - Collaborative Vision-based Perception, Towards Intellingent Robotic Teams (SNF) 


\title{
Distributed Variable-Baseline Stereo SLAM from two UAVs
}

\author{
Marco Karrer and Margarita Chli \\ Vision for Robotics Lab, ETH Zurich, Switzerland
}

\begin{abstract}
Visual-Inertial Odometry (VIO) has been widely used and researched to control and aid the automation of navigation of robots especially in the absence of absolute position measurements, such as GPS. However, when the observable landmarks in the scene lie far away, as in highaltitude flights for example, the fidelity of the metric scale estimate in VIO greatly degrades. Aiming to tackle this issue, in this work, we utilize the virtual stereo setup formed by two Unmanned Aerial Vehicles (UAVs), equipped with one camera and one Inertial Measurement Unit (IMU) each, exploiting their view overlap and relative distance measurements between them using onboard Ultra-Wideband (UWB) modules to enable collaborative VIO, In particular, we propose a decentralized collaborative estimation scheme, where each agent holds its own local map, achieving a low pose estimation latency, while ensuring consistency of each agents' estimates via consensus-based optimization. Following a thorough evaluation in photorealistic simulations, we demonstrate the effectiveness of the approach at high-altitude flights of up to $160 \mathrm{~m}$, going significantly beyond the capabilities of state-of-the-art VIO methods. Finally, we show the advantage of actively adjusting the baseline on-the-fly over a fixed, target baseline, resulting in a significant reduction of the estimation error.
\end{abstract}

Video - https : //youtu .be/SdL4 Jb-BQ28

\section{INTRODUCTION}

The estimation of a robot's motion within a previously unseen workspace is a key skill for autonomous robot navigation. While GPS is of tremendous help towards achieving this goal, its availability and quality are still limited, e.g. indoors or in valleys, hence in general, strong reliance on GPS can be a critical limitation. Addressing this issue, research into Simultaneous Localization And Mapping (SLAM) has received quite some attention as it enables ego-motion estimation using only the sensors carried onboard the robot. Thanks to their complementary nature as well as their low cost and power consumption, cameras and IMUs have been explored extensively to develop robust and accurate state estimation by the means of VIO, such as in [2], [16], [22], which quickly became a standard for autonomous navigation of UAVs [20]. While VIO techniques have successfully been applied in situations, where the scene is in close proximity to the camera (e.g. in autonomous driving, in low-altitude flights or operations indoors), in scenarios where the scene is further away from the camera, such as at high-altitude flights, VIO methods experience difficulties to establish similar levels of accuracy.

As all VIO methods rely on the triangulation of scene landmarks, the scene depth estimates become more uncertain the smaller the triangulation angle gets. For monocular $\mathrm{VIO}$,

This research was supported by the Swiss National Science Foundation (SNSF, Agreement no. PP00P2_183720) and NCCR robotics

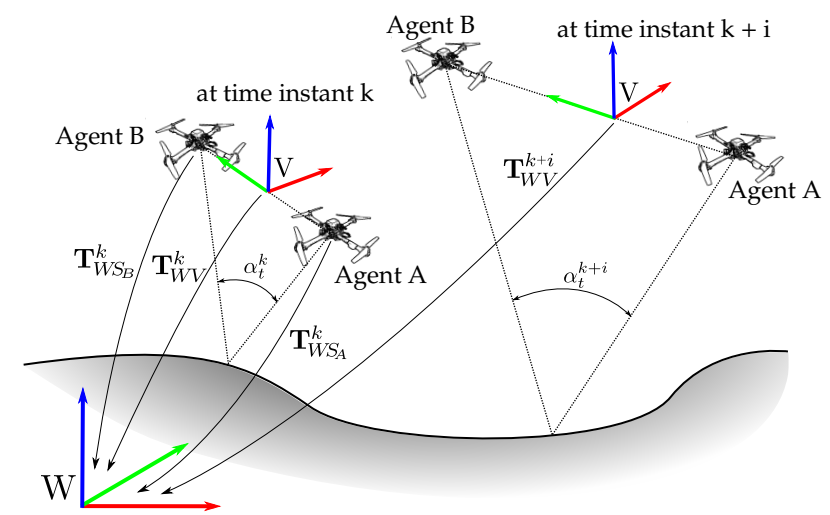

Fig. 1: Illustration of the virtual stereo setup with two UAV agents. Using the desired location of the virtual stereo center $V$ and a target triangulation angle $\alpha_{t}$, the agents' required poses are computed to enable robust ego-motion and scene estimates in the proposed distributed, collaborative framework.

this angle is determined by the camera motion and the scene depth. As the overall scale of monocular VIO conceptually, is determined via the integration of IMU measurements, with increasing scene depth, a larger range of motion is required in order to obtain a large enough triangulation angle. This leads to the integration of more IMU/readings, which at some point results in a numerically weakly constrained scale. The same effect can be observed when using stereo cameras, where the fixed baseline dictates the maximum depth that can be reliably detected [7] and thus, limits the effectiveness of the imposed constraints on the scale.

Inspired by the idea in [1] of using two UAVs equipped each with a camera as a virtual stereo camera rig, in this work we propose a complete system fusing the visual data from two agents along with relative distance measurements to collaboratively estimate the poses of the cameras. In order to allow for low-latency pose estimation of the two agents that is robust against network delays, we propose an optimization based, decentralized architecture allowing each agent to hold its own estimate of the map, while ensuring consistency across the estimation processes onboard each agent. In brief, the main contributions of this work are the following:

- a novel, real-time sensor fusion framework combining monocular-inertial data from two UAVs, and relative distance measurements between them, enabling a reliable pose estimation even at high altitudes,

- a thorough evaluation of the proposed system on photorealistic simulations, demonstrating clear improvements over state-of-the-art stereo-inertial methods, and

- demonstration of the advantage of actively controlling the baseline of the two UAV stereo setup by the means of a simple, high-level formation controller. 


\section{RELATED WORK}

Alongside the emergence of vision based SLAM for single robots [2], [16], [18], [22], research into multi-robot systems has been attracting increasing attention recently. The collaborative frameworks proposed in [5] and [11] demonstrate global mapping from Keyframe (KF) data obtained from multiple UAVs. Other systems aim to distribute the parts within the [SLAM estimation process across the agents and a cental server [13], [23], [25], promising to reduce the computational load for the agents and to make map data generated from each agent available to the rest of the robotic team. In order to enable such systems to scale up to larger teams of robots, research effort has been aiming at avoiding a central server entity and performing all operations in a distributed fashion instead. To this end, many works focus on optimizing a specific aspect of the multi-agent system for a distributed setup, such as place recognition [4], robustness [27] or data exchange [3].

The problem of degrading VIO quality at high altitudes has recently received some attention. Using spline-based scale initialization and selecting only highly informative IMU readings, the work in [17] demonstrates remarkable VIO results at altitudes up to $50 \mathrm{~m}$. Nonetheless, with the IMU being the only source of information for the scale, the approach remains prone to high scale uncertainty. Other methods deal with creating a stereo baseline that is as large as possible by placing the two cameras at the tips of a fixedwing aircraft [7], [8], which still bears the limitation of the aircraft's dimensions. Attempting to push the robustness of stereo-based scale estimation further, the works in [1] and [10] investigate the estimation of the relative transformation between two independently moving UAVs equipped with monocular cameras exploiting of their (relative) motion and view overlap. However, as with [17], these methods solely rely on motion (i.e. IMU readings) to estimate the scale, hence suffering from the same limitations as monocular VIO.

Taking inspiration from [1], in this paper, we expand each agent's sensor-suite with an additional UWB module, enabling relative distance measurements between the agents, providing the scale of the estimates. With this virtual stereo setup, we propose a collaborative framework to perform VIO, which permits the active control of the extent of the stereo baseline adapting it to the current scene depth. In contrast to other collaborative estimators with tight collaboration on the front-end, such as [28], we propose a decentralized architecture allowing for low-latency pose estimation even in the presence of network delays and fitting well within the bandwidth limitations of a standard WiFi module.

\section{METHOD}

\section{A. Notation}

Throughout this work, we use small bold letters (e.g. a) to denote vector values, capital bold letters (e.g. A) to denote matrices and plain capitals (e.g. $A$ ) denote coordinate frames. To indicate a submatrix formed by the rows $r_{i}$ to $r_{j}$ and the columns $c_{l}$ to $c_{k}$ of $\mathbf{A}$ we use the notation
$\mathbf{A}\left[r_{i}, r_{j} ; c_{l}, c_{k}\right]$ with a 1-based indexing. A vector $\mathbf{x}$ expressed in the coordinate frame $A$, is denoted as ${ }_{A} \mathbf{x}$. Rigid body transformations from coordinate frame $B$ to coordinate frame $A$ are denoted by $\mathbf{T}_{A B}$, comprising the translational part of the transformation $\mathbf{p}_{A B}$ and the rotational part $\mathbf{q}_{A B}$. The concatenation of two quaternions $\mathbf{q}_{1}$ and $\mathbf{q}_{2}$ is denoted by $\mathbf{q}_{1} \circ \mathbf{q}_{2}$, whereas the rotation of vector $\mathbf{v}$ by a quaternion is denoted by $\mathbf{q}(\mathbf{v})$. Values that correspond to a prediction are indicated with $\hat{r}$, whereas measurements are denoted

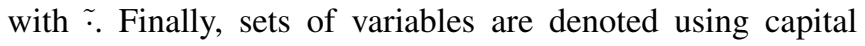
calligraphic letters (e.g. $\mathcal{A})$.

\section{B. Estimator Overview}

The proposed system is designed such that two identical instances are running onboard the two agents, communicating with each other over a wireless connection. The functionality of each instance, as shown in Figure 2, is partitioned into three main threads: tracking, mapping and optimization.

The tracking thread is responsible for estimating the framewise pose by localizing against the current state of the 3D Map Points (MPs). For robust and computationally inexpensive tracking, we fuse the visual observations along with the IMU readings in an Extended Kalman Filter (EKF) Motivated by the fact that the $[\mathrm{KF} \mid$ poses serve as base-poses in the spline based back-end optimization, the current frame is chosen to be a $\mathrm{KF}$ if the time difference between the current frame and the last $\mathrm{KF}$ is larger than a threshold. For every created $\mathrm{KF}$ on an agent, the relevant information (i.e. the $2 \mathrm{D}$ feature locations and their corresponding BRISK features [15] as well as the KF s pose information) are sent to the other agent.

In the mapping thread, the creation of new 3D MPS is attempted between the newest native $\mathrm{KF}$ and a subset of existing KFs. In order to minimize the book-keeping necessary for map maintenance and to avoid complex synchronization across the agents, the system is designed such that each agent holds its own, independent set of MPs Besides the creation of the new MPs, the mapping thread is also responsible for maintaining a rough approximation of the uncertainties of the MPs and establishing the visual correspondences between the non-native $\mathrm{KFs}$ and the MPs

While the initialization and uncertainty estimation of the MPs are carried out by the mapping thread, the position of the MPs are updated as a result of the nonlinear optimization performed by the optimization thread, using a sliding window-based, nonlinear optimization, fusing the UWB information along with the visual correspondences and optimizes both the $\mathrm{KF}$ poses as well as the $\mathrm{MP}$ positions. As each agent estimates its own map-instance, we make use of an asynchronous Alternating Direction Method of Multipliers (ADMM) -variant as proposed by [21] in order ensure consistency across the two agents. By the means of pose consensus, enforced by exchanging a set of dual variables, the approach ensures that both agents converge to a common trajectory estimate. The rest of this section discusses the main modules involved in the proposed system. 


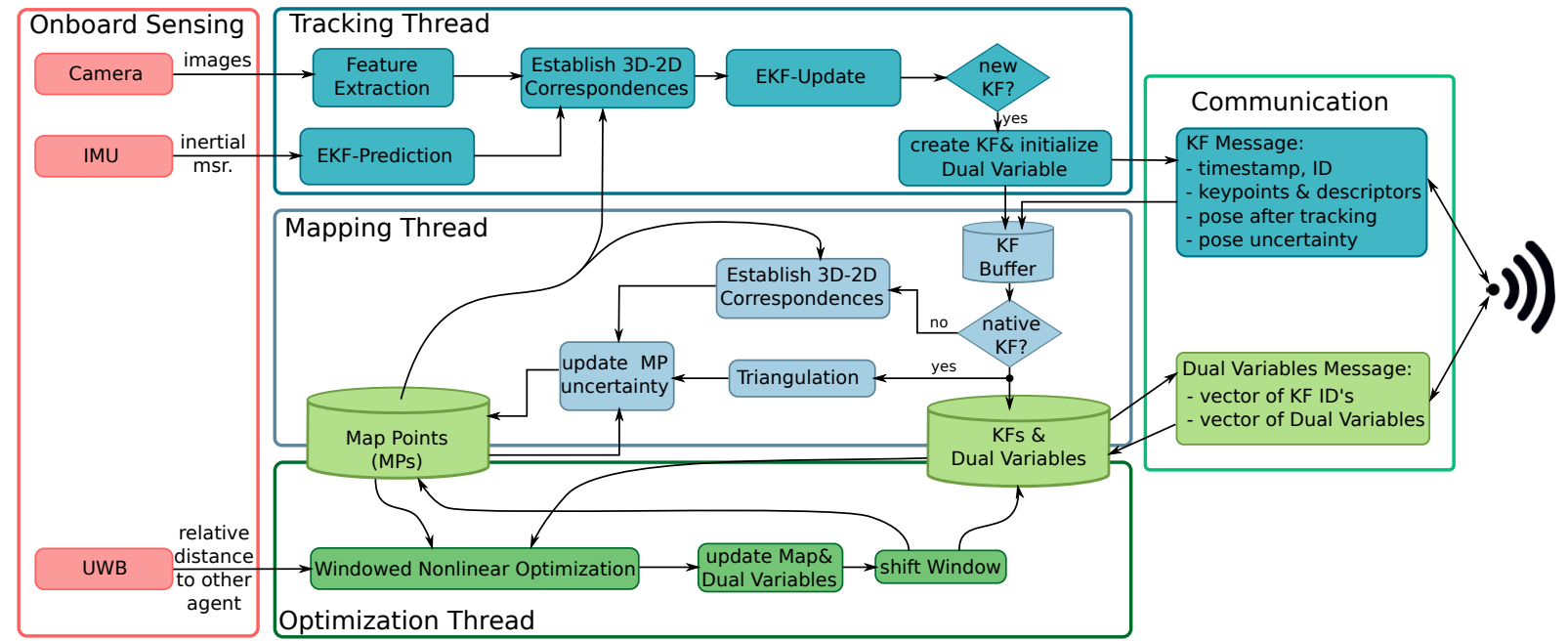

Fig. 2: Schematic of the processes running on each of the two agents for the proposed system mainly comprising of frame-wise tracking, mapping and non-linear optimization. Consistency between the two agents is achieved by communicating KF data and Dual Variables associated to the KF poses via wireless communication.

In order to initialize the system, in the beginning we perform brute force based structure from motion approach on one of the agents and scale the graph with the corresponding $\mathrm{UWB}$ measurements. The result is then communicated to the other agents and the system transitions into the normal operation mode.

\section{EKF-based Pose Tracking}

In order to allow for smooth and robust estimation of the robot's pose, we designed an EKF to fuse the IMU information along with the visual observations of the camera frames. As the MPs have a given position (estimated in the optimization thread) with an associated uncertainty (estimated in the mapping thread) and are, therefore, not considered in the filter, allowing for a compact state vector and lower computational complexity. Cross-correlations between the MPs. however, are ignored leading to a conservative estimate of the state covariance. (i.e. larger than it otherwise would be). As this EKF is intended to guide the search for data association and to obtain a locally smooth pose estimate by incorporating the IMU information, a conservative covariance estimate does not restrict the capabilities of the approach.

The EKF state $\mathcal{X}_{t r}$ is chosen as follows:

$$
\mathcal{X}_{t r}:=\left[\begin{array}{llllll}
\mathbf{q}_{W M} & \mathbf{q}_{M S} & \mathbf{p}_{M S} \mathbf{v} & \mathbf{b}_{a} & \mathbf{b}_{\omega}
\end{array}\right],
$$

where $\mathbf{q}_{W M}$ denotes the rotation (in quaternion form) of this agent's map origin into the gravity-aligned world frame, $\left[\mathbf{q}_{M S}, \mathbf{p}_{M S}\right]$ corresponds to the pose of the IMU frame in the map, ${ }_{S} \mathbf{v}$ denotes the robocentric velocity of the IMU frame, and $\mathbf{b}_{a}$ and $\mathbf{b}_{\omega}$ denote the accelerometer- and the gyroscope biases, respectively. The covariance associated to the state $\mathcal{X}_{t r}$ is denoted as $\boldsymbol{\Sigma}_{t r}$. While rotations are parameterized as quaternions, the gravity rotation $\left(\mathbf{q}_{W M}\right)$ has only two Degree-of-freedom (DoF) (roll and pitch), whereas the pose $\left(\mathbf{q}_{M S}\right)$ has 3 DoF, resulting in $\boldsymbol{\Sigma}_{t r} \in \mathbb{R}^{17 \times 17}$. As commonly applied, e.g. as in [2], we use the IMU measurements for the state propagation. The acceleration ${ }_{S} \tilde{\mathbf{a}}_{S}$ and gyroscope ${ }_{S} \tilde{\boldsymbol{\omega}}_{W S}$ measurements are modeled to be the true acceleration
${ }_{S} \mathbf{a}_{S}$ and rotational velocity ${ }_{S} \boldsymbol{\omega}_{W S}$ affected by both noise and additive biases:

$$
\begin{gathered}
\tilde{\mathbf{a}}_{S}={ }_{S} \mathbf{a}_{S}+\mathbf{b}_{a}+\mathbf{w}_{a} \\
{ }_{S} \tilde{\boldsymbol{\omega}}_{W S}={ }_{S} \boldsymbol{\omega}_{W S}+\boldsymbol{b}_{\omega}+\mathbf{w}_{\omega},
\end{gathered}
$$

where $\mathbf{w}_{a}, \mathbf{w}_{\omega}$ are zero-mean Gaussian noise variables acting on the acceleration and the rotational velocity measurements, respectively. The time variations of the IMU bias variables are modeled as white Gaussian noise processes. Using an Euler forward-integration scheme, we arrive at the following discrete-time state prediction model:

$$
\begin{aligned}
\hat{\mathbf{q}}_{W M}^{k+1} & =\mathbf{q}_{W M}^{t} \\
\hat{\mathbf{q}}_{M S}^{k+1} & =\mathbf{q}_{M S}^{k} \boxplus\left(\Delta t_{S} \boldsymbol{\omega}_{W S}^{k}\right) \\
\hat{\mathbf{p}}_{M S}^{k+1} & =\mathbf{p}_{M S}^{k}+\Delta t \mathbf{q}_{M S}^{k}\left({ }_{S} \mathbf{v}_{S}^{k}\right) \\
{ }_{S} \hat{\mathbf{v}}_{S}^{k+1} & ={ }_{S} \mathbf{v}_{S}^{k}+\Delta t\left(\left(\mathbf{q}_{W M}^{k} \circ \mathbf{q}_{M S}^{k}\right)^{-1}\left({ }_{W} \mathbf{g}\right)+\right. \\
& \left.{ }_{S} \mathbf{a}_{S}-\left({ }_{S} \boldsymbol{\omega}_{W S}^{k}\right)^{\times}{ }_{S} \mathbf{v}_{S}^{k}\right) \\
\hat{\mathbf{b}}_{a}^{k+1} & =\mathbf{b}_{a}^{k} \\
\hat{\mathbf{b}}_{g}^{k+1} & =\mathbf{b}_{g}^{k} .
\end{aligned}
$$

The boxplus operator in Eq. (5) is a generalization of the addition to quantities which are not in a vector space [6]. A concrete definition of the operator for our quaternion-based implementation can be found in [12]. The term $\left({ }_{S} \omega_{W S}^{k}\right)^{\times}$ denotes the skew-symmetric matrix constructed from the tuple ${ }_{S} \boldsymbol{\omega}_{W S}^{k}$ and ${ }_{W} \mathbf{g}$ denotes the gravity vector expressed in the inertial frame. The state covariance gets forward predicted using the standard EKF formulation constructed out of Eq. (4)-(9). For the state update, we use projective correspondences to the most recent available estimates of the MPs as measurements. In order to establish these correspondences we use the predicted pose $\hat{\mathbf{q}}_{M S}^{k+1}, \hat{\mathbf{p}}_{M S}^{k+1}$ and project all MPs into the camera frame:

$$
\mathbf{z}_{\text {proj }_{i}}=\pi\left(\mathbf{T}_{C S} \hat{\mathbf{T}}_{M S M}^{-1} \mathbf{m}_{i}\right)
$$

where the function $\pi(\cdot)$ denotes the projection function (including distortion). The transformation $\mathbf{T}_{C S}$ between the 
IMU and the camera is considered to be known and can be obtained from calibration. To perform the data association, we match the $2 \mathrm{D}$ keypoint descriptors in a small radius around the projection $\mathbf{z}_{\text {roj }_{i}}$ against the $\mathrm{MP}$ s descriptor, followed by a 3D-2D-RANSAC [14] based outlier rejection Using the remaining inlier correspondences, the reprojection residuals are given by

$$
\mathbf{y}_{i, j}=\tilde{\mathbf{z}}_{j}-\mathbf{z}_{\text {rroj }_{i}}
$$

where $\tilde{\mathbf{z}}_{j}$ is the pixel coordinates of the $2 \mathrm{D}$ keypoint $j$ in the image space that was associated with $\mathrm{MP} i$. For the EKF update, we stack all the individual residuals $\mathbf{y}_{i, j}$ together to a residual vector $\mathbf{y}$ and compute the corresponding state Jacobian H. As the MPs are not part of the EKF state, we project the estimated MP uncertainty $\Sigma_{m_{i}}$ onto the image plane, in order to inflate the measurement uncertainty:

$$
\mathbf{R}_{i}=\mathbf{J}_{\mathbf{m}_{i}} \boldsymbol{\Sigma}_{m_{i}} \mathbf{J}_{\mathbf{m}_{i}}^{T}+\operatorname{diag}\left\{\sigma_{o b s}^{2}, \sigma_{o b s}^{2}\right\}
$$

where $\mathbf{J}_{\mathbf{m}_{i}}$ is the part of the residual Jacobian w.r.t. the $\mathrm{MP}$ position and $\sigma_{o b s}$ is the keypoint uncertainty, which in our case is only dependent on the octave that this keypoint was detected. Using the inflated measurement uncertainties, the tracking state and its covariance are updated using the standard EKF equations.

\section{Mapping}

The mapping thread of the proposed pipeline is responsible for both the generation of new MPs as well as the maintenance of a rough estimate of their uncertainties. The initialization of new MPs is performed by triangulating 2D2D correspondences between the most recent native KF (target) and the KFs that are already in the Map (candidates). In order to limit the computational complexity, we select a subset of candidate KFs to match against, based on the relative viewpoint with respect to the target $\mathrm{KF}$, while favoring non-native over native candidates.s. Using the set of chosen candidate $\mathrm{KFs}$, we sequentially perform brute-force descriptor matching followed by as 2D-2D RANSAC-based outlier rejection. The inlier-correspondences are triangulated using a SVD-based linear triangulation in order to obtain the 3D position of the new MPs ${ }_{M} \mathbf{m}_{i, j}$ using the KFs pose estimates $\mathbf{T}_{M S_{i}}$ and $\mathbf{T}_{M S_{j}}$. Using the estimated covariances of the $\mathrm{KF}$ poses $\boldsymbol{\Sigma}_{\mathbf{T}_{M S_{i}}}, \boldsymbol{\Sigma}_{\mathbf{T}_{M S_{i}}}$, we estimate the uncertainty $\boldsymbol{\Sigma}_{M} \mathbf{m}_{i, j}$ of the triangulated MP as:

$$
\boldsymbol{\Sigma}_{M} \mathbf{m}_{i, j}=\left(\mathbf{J}_{i, j} \mathbf{W}_{i, j} \mathbf{J}_{i, j}\right)^{-1}[1,3 ; 1,3],
$$

where $\mathbf{J}_{i, j}$ is the Jacobian of the re-projected MPs and the poses. The full form of the Jacobian $\mathbf{J}_{i, j}$ can be found in [12]. The information matrix $\mathbf{W}_{i, j}$ has a block-diagonal form and is given by:

$$
\mathbf{W}_{i, j}=\operatorname{diag}\left\{\sigma_{\text {obs }}^{-2} \cdot \mathbf{I}_{2 \times 2}, \boldsymbol{\Sigma}_{\mathbf{T}_{M S_{i}}}^{-1}, \boldsymbol{\Sigma}_{\mathbf{T}_{M S_{j}}}^{-1}\right\} .
$$

In order to maintain the approximation of the MPs uncertainties, we run an independent EKF per MP While this is a strong simplification, e.g. it neglects the cross-correlations between MPs this sacrifice was made in favour of a computationally lightweight implementation. It is worth noting that this approximation only affects the uncertainty estimation, as the MPs positions are updated in the optimization thread. We model the dynamic of the MPs as random walk in order to account for positional changes e.g. caused by the optimization and propagate the covariance as follows:

$$
\hat{\boldsymbol{\Sigma}}_{\mathbf{m}_{i}}^{k+1}=\boldsymbol{\Sigma}_{m_{i}}^{k}+\left(\left\|_{S} \mathbf{m}_{i}\right\| \cdot \sigma_{m}\right)^{2} \cdot \mathbf{I}_{3 \times 3}
$$

where $\sigma_{m}$ is the noise parameter, which was chosen to be 0.005 and $\left\|_{S_{k}} \mathbf{m}_{i}\right\|$ denotes the depth of the MP seen in the most recent $\mathrm{KF}$. In order to update the estimated covariance, we utilize the reprojection error $\mathbf{y}_{i}$ of the established 2D3D correspondences of both native- and non-native KFs. As the MPs filters do not include the poses of the KFs, we perform a similar operation as in Eq. 12 to obtain an inflated measurement noise:

$$
\mathbf{R}_{\mathbf{p}_{y, i}}=\mathbf{H}_{\mathbf{T}_{M S}} \hat{\boldsymbol{\Sigma}}_{\mathbf{T}_{M S}} \mathbf{H}_{\mathbf{T}_{M S}^{T}}^{T}+\sigma_{o b s}^{2} \cdot \mathbf{I}_{2 \times 2}
$$

where the $\mathbf{H}_{\mathbf{T}_{M S}}$ denotes the Jacobian of $\mathbf{y}_{i}$ with respect to $\mathrm{KF}$ pose $\mathbf{T}_{M S}$. Again, using the inflated measurement noise, we use the standard EKF equations to update the statecovariance.

\section{E. Optimization Back-End}

The optimization back-end constitutes a core element of the system and is responsible for the fusion of the visual information and the UWB distance measurements, which ultimately provides the metric scale in the system. In order to allow each agent to have a complete map, while avoiding a complex synchronization effort across the agents, we propose to allow each agent to have its independent set of MPs, but for consistency we enforce the trajectories to be identical across both agents by introducing the following constraints:

$\mathbf{T}_{M S_{A, A}}^{i}=\mathbf{T}_{M S_{A, B}}^{i}, \mathbf{T}_{M S_{B, A}}^{i}=\mathbf{T}_{M S_{B}, B}^{i}, \forall i \in[1, N-l]$.

We denote the pose of agent $i$ as estimated by agent $j$ using $\mathbf{T}_{M S_{i, j}}$. The parameter $l \geq 0$ is used to represent a lag between the creation of a $\mathrm{KF}$ on one agent until it is available to the other one, e.g. caused by network delays. Note that for every such introduced constraint on every agent $i$ a dual variable $\mathbf{z}_{i}$ is introduced as shown in [12], [21]. Therefore, the optimization variables for agent $i$ are given by:

$$
\mathcal{X}_{i}:=[\underbrace{\mathbf{T}_{M S_{i}}^{1}, \cdots \mathbf{T}_{M S_{i}}^{N}}_{\text {native KFs }}, \underbrace{\mathbf{T}_{M S_{\ulcorner}}^{1}, \cdots, \mathbf{T}_{M \Gamma_{i}}^{N-l}}_{\text {non-native KFs }}, \underbrace{{ }_{M} \mathbf{m}^{1}, \cdots,{ }_{M} \mathbf{m}^{M_{i}}}_{\text {Map Points }}], i \in\{A, B\},
$$

where the notation $\ulcorner i$ indicates the opposite index, i.e. $i=$ $A \Rightarrow\ulcorner i=B$. Over these variables, we can define the optimization objective as:

$f\left(\mathcal{X}_{i}\right):=\sum_{k \in \mathcal{K}} \sum_{j \in \mathcal{M}(k)} \delta_{c}\left(\mathbf{y}_{k, j}^{T} \mathbf{W}_{r}^{k, j} \mathbf{y}_{k, j}^{T}\right)+\sum_{u \in \mathcal{D}} \delta_{c}\left(\frac{1}{\sigma_{d}^{2}} e_{d}^{u 2}\right)$

where the set $\mathcal{K}$ indicates all $\mathrm{KFs} i$, native and non-native, that are currently inside the sliding window and accordingly, $\mathcal{M}(k)$ indicates all the MPs that are visible in KF $k$. The function $\delta_{c}(\cdot)$ denotes a robust loss function, here the 
Cauchy loss function, introduced to reduce the influence of outliers. The terms $\mathbf{y}_{k, j}$ are the reprojection residuals as defined in Eq. (10) and (11) with the corresponding weights given by $\mathbf{W}_{r}^{k, j}=\sigma_{\text {obs }}^{-2} \cdot \mathbf{I}_{2 \times 2}$. The set of relative distance measurements with standard deviation $\sigma_{d}$ between the two agents is denoted by $\mathcal{D}$, while the corresponding residual terms are given by

$e_{d}^{u}:=\left\|\mathbf{q}_{M S_{A}}(t)\left(\mathbf{p}_{U_{A}}\right)+\mathbf{p}_{M S_{A}}(t)-\mathbf{q}_{M S_{B}}(t)\left(\mathbf{p}_{U_{B}}\right)-\mathbf{p}_{M S_{B}}(t)\right\|-d_{\text {meas }}^{u}$,

where $d_{\text {meas }}^{u}$ corresponds to the distance measurement taken at time $t$, and $\mathbf{p}_{U_{i}}, i \in\{A, B\}$ is the UWB antenna offset expressed in the corresponding IMU frame. The poses $\mathbf{p}_{M S_{i}}(t), \mathbf{q}_{M S_{i}}(t), i \in\{A, B\}$ are computed using a cubic Z-spline based interpolation [24] using the KFs neighbouring the timestamp $t$ as base poses. Extending the optimization objective as introduced in Eq. (19) with some additional error terms $\mathbf{e}_{c}$ accounting for the constraints in Eq. (17), we arrive at the formulation for the state update of each agent

$$
\mathcal{X}_{i}^{k+1}=\underset{\mathcal{X}_{i}}{\arg \min } f\left(\mathcal{X}_{i}\right)+\sum_{j=1}^{N}\left\|\mathbf{e}_{c}^{i, j}\right\|^{2}+\sum_{j=1}^{N-l}\left\|\mathbf{e}_{c}^{\ulcorner i, j}\right\|^{2}
$$

The consensus-error terms $\mathbf{e}_{c}$ essentially penalize differences between the local estimates from one agent's KF poses and the ones estimated by its neighbour. While the update in Eq. (21) is performed iteratively, after each optimization step the dual variables associated to the constraints Eq. (17) are updated and communicated to the neighbouring agent. For a detailed mathematical formulation of the error terms and the dual variable update scheme, the reader is referred to [12].

\section{F. Active Baseline Controller}

In order to control the relative distance between the UAV agents, a high-level controller was designed such that the agents' baseline forms a virtual stereo camera as illustrated in Figure 1. For simplicity, we assume that the monocular cameras are mounted at an identical viewing angle onboard each agent and that aligning the agents along their $\mathrm{X}$-axes results in a valid stereo configuration. The translational part for the virtual stereo camera system is given by the average of the two agents' poses as $\mathbf{p}_{W V}^{k}=0.5 \cdot\left(\mathbf{p}_{W S_{A}}^{k}+\mathbf{p}_{W S_{B}}^{k}\right)$. In order to obtain the yaw angle, we first project the relative baseline onto the $\mathrm{X}-\mathrm{Y}$ plane of the inertial frame $\mathbf{p}_{\text {proj }}=\left(\mathbf{p}_{W S_{B}}^{k}-\mathbf{p}_{W S_{A}}^{k}\right)[1,2]$. From that we can compute the resulting yaw angle formed coordinate system $V$ as:

$$
\psi_{W V}=\operatorname{atan} 2\left(\mathbf{p}_{\text {proj }}[2], \mathbf{p}_{\text {proj }}[1]\right)+\pi / 2,
$$

Using the desired triangulation angle $\alpha_{t}$ (here set to $10^{\circ}$ ) and the scene depth $d_{s}$, the resulting baseline of the virtual stereo camera is given by

$$
b_{V}=2 \cdot \tan \left(\alpha_{t} / 2\right) \cdot d_{s} .
$$

Hence, the resulting agents' target translations are given by

$$
\mathbf{p}_{W S_{A / B}}=\mathbf{p}_{t}+\mathbf{q}_{\psi_{t}}\left(\left[\begin{array}{lll}
0 & \mp 0.5 \cdot b_{t} & 0
\end{array}\right]^{T}\right),
$$

where the minus sign corresponds to agent $\mathrm{A}$ and the plus sign to agent $\mathrm{B}$, assuming that agent $\mathrm{A}$ acts as the

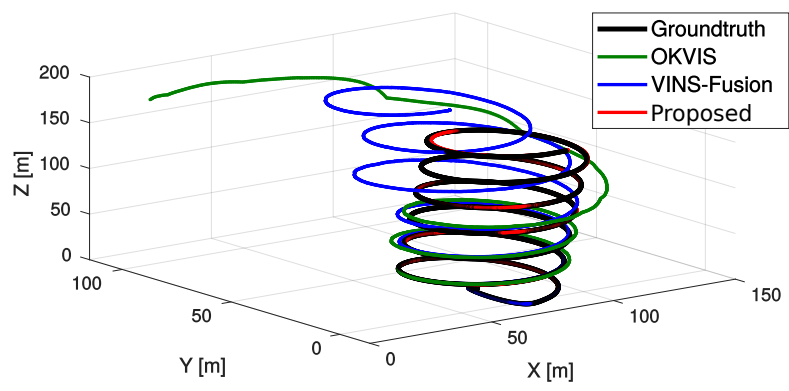

Fig. 3: Comparison of the proposed approach against state-of-the-art stereoinertial methods for a trajectory with increasing scene depth. Both single agent methods start to diverge with increasing altitude.

right camera in the virtual stereo setup. The values $\mathbf{p}_{t}, \mathbf{q}_{\psi_{t}}$ corresponds to the target pose of the virtual stereo camera frame $V$ and $b_{t}$ is the corresponding baseline. The target yaw angles of the agents are set to the target yaw angle $\psi_{t}$ of the virtual stereo camera. The computed target poses for both agents are fed to the MPC-based position controller running on each agent [9], while linearly interpolating between the current and the target pose if the target set-points are too far away.

\section{EXPERIMENTAL EVALUATION}

\section{A. Photo-Realistic Synthetic Datasets}

Performing experiments with two UAVs flying relatively close to each other at high altitudes is extremely challenging as at that altitude the pilot has very limited visual understanding of the motion of the UAVs, posing a significant risk of losing control of the aircraft. As result, we instead simulate the sensor data using the simulation pipeline proposed in [26]. The images are rendered with a resolution of $480 \times 752$ at $20 \mathrm{~Hz}$ and the IMU data has a rate of $200 \mathrm{~Hz}$. The UWB-distance measurements are simulated by computing the ground-truth relative distance between the UAVs and disturb it with gaussian noise. The UWB-data is simulated at $60 \mathrm{~Hz}$ with a noise level of $0.1 \mathrm{~m}$ standard deviation.

In order to control the relative pose between the two UAVs we employ the control strategy as outlined in Section III-F while the estimated scene depth is obtained by computing the median depth from a rendered depth image. We use four different scenes (Scene 1-4), which can be seen in the accompanying video 1

\section{B. Comparison to Visual-Inertial SLAM at higher altitudes}

In this section, we provide a comparison of the proposed approach against the two highest performing stateof-the-art stereo VIO methods that are publicly available, namely VINS-Fusion [22] and OKVIS [16], to test their accuracy of estimates at relatively large scene depths. In a first experiment, we investigate the effect of the scene depth by generating spiral-shaped trajectories with a radius of $25 \mathrm{~m}$ and an altitude increase of $25 \mathrm{~m}$ per turn up to a height of approximately $160 \mathrm{~m}$. The fixed stereo camera baseline for VINS-Fusion and OKVIS was chosen to be

https://youtu.be/SdL4Jb-BQ28 


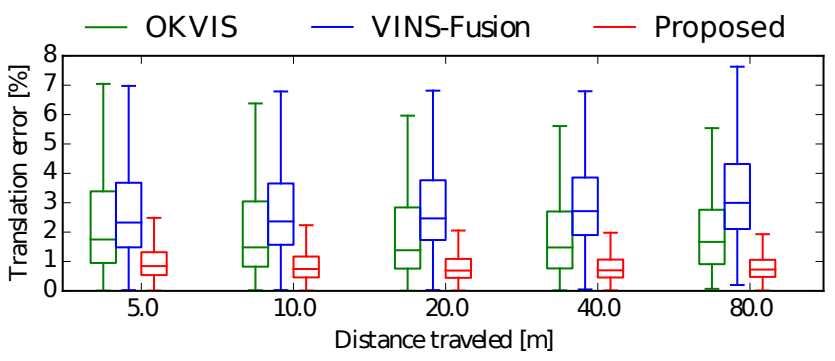

Fig. 4: Comparison of the relative odometry error between state-of-the-art stereo VIO methods and the proposed approach. The reported errors are obtained by computing the error statistics over all datasets over 3 runs.

$0.22 \mathrm{~m}$, which was chosen to be twice the size of the sensor proposed in [19]. Such an example trajectory along with the aligned estimates of both our system and the stereo-inertial estimators is shown in Figure 3. Initially, all the estimates are close to the ground-truth, however, both estimates of VINSFusion as well as OKVIS start to diverge with increasing altitude, while the estimate of the proposed system remains close to the ground-truth trajectory. We observed the same tendency throughout the different scenes and identified the degradation of the metric scale as the leading cause of this behavior.

In order to allow for a quantitative comparison between our proposed approach to the two state-of-the-art stereo VIOs two different trajectories at altitudes ranging between 15-35 meters for each of the Scenes 1-3 were rendered. For the proposed method, the baseline between the two agents is chosen to be roughly $2 \mathrm{~m}$, while this is allowed to fluctuate slightly without considering the particular scene depth. The comparison between the relative odometry errors of the stereo VIO methods and the proposed method is shown in Figure 4. As it can be observed, compared to the existing VIO methods, the proposed system has both a lower median error as well as smaller fluctuations in the statistics, as expected, due to the fact that the selected baseline should exhibit more favorable behavior for the overall scene depth. Between the two existing stereo VIOs, OKVIS performs slightly better than VINS-Fusion on the evaluated datasets, whereas to a large extent the increased error of the latter can be traced back to an increased yawdrift. Note that the evaluated datasets are closer to the intended use-cases of the proposed system, i.e. scene depths above $10 \mathrm{~m}$, than the VIOs, which are generally designed and evaluated on datasets closer to the ground or indoors. Hence, we do not claim to outperform these systems in the general case, however, we can show the advantage of the proposed approach already at flying altitudes significantly below the heights where the stereo VIO systems start to fail.

\section{Fixed vs. Adaptive Virtual Stereo Baseline}

In this section, we evaluate the influence of actively adapting the virtual stereo baseline, i.e. a fixed $\alpha_{t}$, between the two UAVs against maintaining a fixed target distance between the two aircraft. As this generally cannot be achieved using the exact same trajectories, for every dataset, we create two versions; one using a fixed baseline of $2 \mathrm{~m}$ and another,

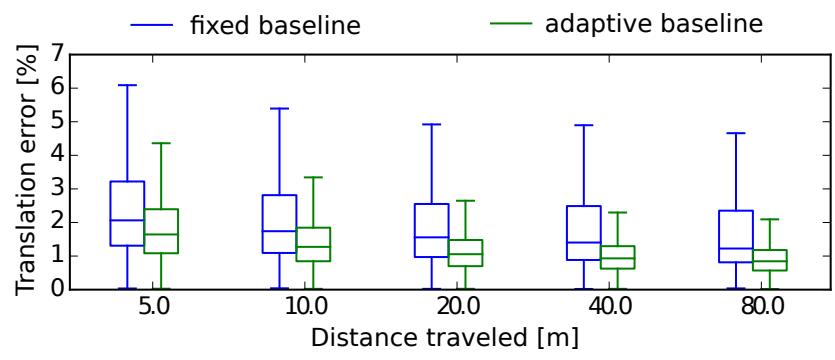

Fig. 5: Comparison of the relative translation error over all runs on all datasets, summing up to an overall trajectory length of $3.4 \mathrm{~km}$.

where the baseline gets constantly adjusted to achieve a triangulation angle $\alpha_{t}$ (of $10^{\circ}$ ). However, the waypoints as well as the simulation parameters are otherwise chosen to be identical. The estimator parameters in both versions are identical. In particular, the $\mathrm{KF}$ interval is set to 0.15 seconds and the trajectory horizon to 5.0 seconds for all the experiments in this section. In total, five datasets are generated on the four scenes, where the waypoints are chosen to follow mostly exploratory paths with some height variation, leading to scene depths ranging between $25-120 \mathrm{~m}$. The resulting error statistics of the obtained odometry estimates for both cases are summarized in Figure 5. As it can be observed, the position drift is significantly reduced when adapting the baseline versus having a fixed baseline. The relative translation error of the fixed baseline approach ranges between $1.8-2.5 \%$, while using an adaptive baseline the error can be reduced to $0.9-1.9 \%$, corresponding to up to a twofold reduction.

\section{CONCLUSiON}

In this work, we present a novel framework using two UAVs, equipped with one IMU and one monocular camera each, while measuring the relative distance between them using an UWB module in order to compute the poses (in six degrees of freedom) for both UAVs in real-time to estimate the scene in a virtual-stereo setup. The pipeline is implemented in a decentralized fashion allowing each agent to hold its own estimate of the map, enabling low-latency pose estimation that is robust against network delays, while consistency across the agents is ensured using a consensusbased optimization scheme. Making use of the virtual stereo camera setup, a simple formation controller, which adjusts the stereo baseline between the two agents adaptively depending on the observed scene depth was designed.

In our experimental analysis using synthetic data, we could demonstrate the ability of the proposed approach to reliably estimate the poses of the agents at high altitudes, where established stereo-inertial methods struggle. The comparison with state-the-art stereo VIO methods demonstrates, that the proposed approach already proves advantageous in terms of estimation accuracy at altitudes marginally higher than $15 \mathrm{~m}$. By deploying the proposed baseline control scheme instead of using a fixed target baseline, a nearly twofold reduction of the pose estimation error is achieved. Finally, given the great promise of the proposed system future work will focus on enabling swift large-scale 3D scene reconstructions. 


\section{REFERENCES}

[1] M. W. Achtelik, S. Weiss, M. Chli, F. Dellaert, and R. Siegwart. Collaborative Stereo. In Proceedings of the IEEE/RSJ Conference on Intelligent Robots and Systems (IROS), 2011.

[2] M. Bloesch, M. Burri, S. Omari, M. Hutter, and R. Siegwart. Iterated extended kalman filter based visual-inertial odometry using direct photometric feedback. The International Journal of Robotics Research, 36(10):1053-1072, 2017.

[3] T. Cieslewski, S. Choudhary, and D. Scaramuzza. Data-efficient decentralized visual SLAM. In Proceedings of the IEEE International Conference on Robotics and Automation (ICRA), pages 2466-2473, 2018.

[4] T. Cieslewski and D. Scaramuzza. Efficient decentralized visual place recognition using a distributed inverted index. IEEE Robotics and Automation Letters, 2(2):640-647, 2017.

[5] C. Forster, S. Lynen, L. Kneip, and D. Scaramuzza. Collaborative Monocular SLAM with Multiple Micro Aerial Vehicles. In Proceedings of the IEEE/RSJ Conference on Intelligent Robots and Systems (IROS), 2013

[6] C. Hertzberg, R. Wagner, U. Frese, and L. Schröder. Integrating generic sensor fusion algorithms with sound state representation through encapsulation of manifolds. Information Fusion, 2011.

[7] T. Hinzmann, C. Cadena, J. Nieto, and R. Siegwart. Flexible Trinocular: Non-rigid Multi-Camera-IMU DenseReconstruction for UAV Navigation and Mapping. In 2019 IEEE/RSJ International Conference onIntelligent Robots and Systems (IROS. IEEE, 2019.

[8] T. Hinzmann, T. Taubner, and R. Siegwart. Flexible Stereo: Constrained, Non-Rigid, Wide-Baseline Stereo Vision for Fixed-Wing Aerial Platforms. In Proceedings of the IEEE International Conference on Robotics and Automation (ICRA), pages 2550-2557, May 2018.

[9] M. Kamel, T. Stastny, K. Alexis, and R. Siegwart. Model Predictive Control for Trajectory Tracking of Unmanned Aerial Vehicles Using Robot Operating System. In A. Koubaa, editor, Robot Operating System (ROS) The Complete Reference, Volume 2. Springer, 2017.

[10] M. Karrer, M. Agarwal, M. Kamel, R. Siegwart, and M. Chli. Collaborative 6DoF Relative Pose Estimation for Two UAVs with Overlapping Fields of View. In Proceedings of the IEEE International Conference on Robotics and Automation (ICRA), pages 6687 - 6693, 2018.

[11] M. Karrer and M. Chli. Towards Globally Consistent Visual-Inertial Collaborative SLAM. In Proceedings of the IEEE International Conference on Robotics and Automation (ICRA), pages 3685 - 3692, 2018

[12] M. Karrer and M. Chli. Distributed Variable-Baseline Stereo SLAM from two UAVs. arXiv:2009.04801, 2020.

[13] M. Karrer, P. Schmuck, and M. Chli. CVI-SLAM - Collaborative Visual-Inertial SLAM. IEEE Robotics and Automation Letters, 3(4):2762-2769, 2018

[14] L. Kneip, D. Scaramuzza, and R. Siegwart. A Novel Parametrization of the Perspective-Three-Point Problem for a Direct Computation of
Absolute Camera Position and Orientation. In Proceedings of the IEEE Conference on Computer Vision and Pattern Recognition (CVPR), 2011.

[15] S. Leutenegger, M. Chli, and R. Siegwart. BRISK: Binary robust invariant scalable keypoints. In Proceedings of the International Conference on Computer Vision (ICCV), 2011.

[16] S. Leutenegger, S. Lynen, M. Bosse, and P. Siegwart, Roland anand Furgale. Keyframe-based visual-inertial SLAM using nonlinear optimization. International Journal of Robotics Research (IJRR), 34(3):314-334, 2017.

[17] T. Liu and S. Shen. High Altitude Monocular Visual-Inertial State Estimation:Initialization and Sensor Fusion. In Proceedings of the IEEE International Conference on Robotics and Automation (ICRA), pages $4544-4551,2017$.

[18] R. Mur-Artal and J. D. Tardós. ORB-SLAM2: An Open-Source SLAM System for Monocular, Stereo and RGB-D Cameras. IEEE Transactions on Robotics (T-RO), 33(5):1255-1262, 2017.

[19] J. Nikolic, J. Rehder, M. Burri, P. Gohl, S. Leutenegger, P. T. Furgale, and R. Siegwart. A synchronized visual-inertial sensor system with FPGA pre-processing for accurate real-time SLAM. In Robotics and Automation (ICRA), 2014 IEEE International Conference on, pages 431-437. IEEE, 2014.

[20] H. Oleynikova, Z. Taylor, A. Millane, R. Siegwart, and J. Nieto. A Complete System for Vision-Based Micro-Aerial Vehicle Mapping, Planning, and Flight in Cluttered Environments. 2019.

[21] Z. Peng, Y. Xu, M. Yan, and W. Yin. ARock: An Algorithmic Framework for Asynchronous Parallel Coordinate Updates. SIAM Journal on Scientific Computing, 38:2851-2879, 2016.

[22] T. Qin, P. Li, and S. Shen. VINS-Mono: A Robust and Versatile Monocular Visual-Inertial State Estimator. IEEE Transactions on Robotics (T-RO), 34(4):1004-1020, 072018.

[23] L. Riazuelo, J. Civera, and J. Montiel. C2TAM: A cloud framework for cooperative tracking and mapping. Robotics and Autonomous Systems (RAS), 62(4):401-413, 2014

[24] B. Sagredo and J. Tercero. Z-splines: moment conserving cardinal spline interpolation of compact support for arbitrarily spaced data. 2003.

[25] P. Schmuck and M. Chli. CCM-SLAM: Robust and Efficient Centralized Collaborative Monocular SLAM for Robotic Teams. Journal of Field Robotics (JFR), 36(4):763-781, 2018.

[26] L. Teixeira, M. R. Oswald, M. Pollefeys, and M. Chli. Aerial SingleView Depth Completion with Image-Guided Uncertainty Estimation. IEEE Robotics and Automation Letters, 5(2):1055-1062, 42020.

[27] H. Zhang, X. Chen, H. Lu, and J. Xiao. Distributed and collaborative monocular simultaneous localization and mapping for multi-robot systems in large-scale environments. International Journal of Advanced Robotic Systems, 15(3):1729881418780178, 2018.

[28] D. Zou and P. Tan. CoSLAM: Collaborative Visual SLAM in Dynamic Environments. In IEEE Transactions on Pattern Analysis and Machine Intelligence (PAMI), 2013. 